\title{
Research Paper: Comparison of the Effect of Cognitive Rehabilitation and Neurofeedback on Sustained Attention Among Elementary School Students With Specific Learning Disorder: APreliminary Randomized Controlled Clinical Trial
}

\author{
Amir Azizi $^{1}$ (1D), Fazlolah Mir Drikvand ${ }^{1 *}$ (D), Mohamad Ali Sepahvani ${ }^{1}$
}

1. Department of Psychology, Faculty of Literature and Human Sciences, Lorestan University, Khoram Abad, Iran

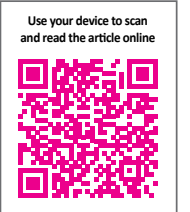

Crtation: Azizi, A., Mir Drikvand, F., \& Sepahvani, M.A. Comparison of the Effect of Cognitive Rehabilitation and Neurofeedback on Sustained Attention Among Elementary School Students With Specific Learning Disorder: A Preliminary Randomized Controlled Clinical Trial. Basic and Clinical Neuroscience, 11(4), 465-472. http://dx.doi.org/10.32598/bcn.11.4.1211.1

\section{(c) (1) (\$)}

Article info:

Received: 06 Sep 2017

First Revision: 20 Oct 2017

Accepted: 10 Feb 2020

Available Online: 01 Jul 2020

Keywords:

Cognitive rehabilitation, Neurofeedback, Sustained attention, Specific learning disorder
A B S T RA C T

Introduction: Sustained Attention (SA) failure is one of the main characteristics of Specific Learning Disorders (SLD). Recent studies have reported a positive effect of Cognitive Rehabilitation (CR) and Neurofeedback (NFB) on SA in SLD. Thus, the effectiveness of CR and NFB is well understood. This preliminary study aimed to compare the effects of CR and NFB on SA among the elementary school students with SLD, using a randomized controlled clinical trial.

Methods: In this preliminary randomized controlled clinical trial, 53 eligible students with SLD (based on DSM-5) within the age range of 7 to 10 years were randomly assigned in the NFB $(n=18), C R(n=18)$, and control group $(n=17)$. All the participants were evaluated for SA using the Continuous Performance Test (CPT), at the time of entry to the study, and one month later. The intervention groups participated in 20 sessions of CR and 20 sessions of NFB, while the control group was evaluated without any intervention.

Results: A total number of 24 boys and 36 girls in four groups $(n=15)$ completed the study. The Mean \pm SD age of the participants in the CR, NFB, and control groups were $8.66 \pm 1.48$ years, $8.40 \pm 1.73$ years, and $8.53 \pm 1.63$ years, respectively. Results showed a significant difference in the variables of the CPT between the study groups $(\mathrm{P}<0.05)$. Also, the CPT scores of the CR group were higher than that of the NFB group $(\mathrm{P}<0.001)$.

Conclusion: This study supports that CR is more effective than NFB on SA in students with SLD.

* Corresponding Author: 


\section{Highlights}

- There is a significant difference in the variables of the CPT between the study groups.

- The CPT scores of the CR group were higher than that of the NFB group.

\section{Plain Language Summary}

Sustained Attention (SA) failure is one of the main characteristics of Specific Learning Disorders (SLD). Recent studies have reported a positive effect of Cognitive Rehabilitation (CR) and Neurofeedback (NFB) on SA in SLD. Thus, the effectiveness of CR and NFB is well understood. The study aimed to compare the effects of CR and NFB on SA among the elementary school students with SLD, using a randomized controlled clinical trial.In this preliminary randomized controlled clinical trial, 53 eligible students with SLD (based on DSM-5) within the age ranged 7-10 years were randomly assigned in the NFB $(n=18), C R(n=18)$, and control group $(n=17)$. All the participants were evaluated for SA using the Continuous Performance Test (CPT), at the time of entry to the study and one month later. The intervention groups participated in 20 sessions of CR and 20 sessions of NFB, while the control group was evaluated without any intervention. Results showed a significant difference in the variables of the CPT between the study groups. Also, the CPT scores of the CR group were higher than that of the NFB group .

\section{Introduction}

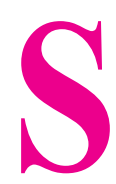

pecific Learning Disorder (SLD) is one of the most common neurodevelopmental disorders among school-aged children (Moll, Kunze, Neuhoff, Bruder, \& Schulte-Korne, 2014). It involves ongoing problems in learning key academic skills, including reading, writing, and math. The prevalence of SLD is $5 \%$ to $15 \%$ in the area of education among elementary school children (DSM-5, American Psychiatric Association, 2013). The students with SLD are more likely to drop out of school (Vogel \& Reder, 1989), remain unemployed (Shapiro \& Lentz, 1991), or even have suicidal thoughts, compared with other students (Daniel et al., 2006).

Sustained Attention (SA) is the ability to direct and focus on a specific stimulus without being distracted for a continuous amount of time (Oken, Salinsky, \& Elsas, 2006; Bergera \& Cassutob, 2014). Learning Disabilities (LD) can coexist with the problems in attention performance that complicates the LD (Sterr, 2004). Evidence shows that children with specific reading disorders have deficits in SA (Duncan et al.,1994). Also, some studies have demonstrated that children with dyslexia exhibit attention deficits (Facoetti et al., 2006; Marzocchi, Ornaghi, Barboglio, 2009). For example, Facoetti and Molteni (2000) showed that children with dyslexia have problems in focusing attention on text and the simultaneous inhibition of distractor stimuli.
In recent decades, a growing interest has been observed in the use of Cognitive Rehabilitation (CR) in the field of executive functioning (Rodríguez-Blanco, Lubrini, Vidal-Mariño, \& Ríos-Lago, 2017) among children and adolescents with LD (Peijnenborgh, Hurks, Aldenkamp, Vles, \& Hendriksen, 2016). CR is a collection of methods designed to increase executive functioning, such as perception, attention, comprehension, learning, memory, problem-solving, and reasoning in people with impairments in these areas (Galletly \& Rigby, 2013). Evidence shows that CR can promote SA in LD (Gray et al., 2012), cerebral palsy (Muriel, Garcia-Molina, Aparicio-Lopez, Ensenat, \& Roig-Rovira, 2014), Alzheimer disease (Kasper, 2015), and multiple sclerosis (Amato et al., 2013; De Giglio et al., 2015).

Neurofeedback (NFB) is another technique used to reduce the symptoms of LD (Mosanezhad Jeddi \& Nazari, 2013). By the regulation of the Electroencephalogram (EEG), functional Magnetic Resonance Imaging (fMRI), and HEG abnormalities, NFB can be a helpful intervention in the treatment of some disorders (Nazari, Querne, De Broca, \& Berquin, 2011). This treatment approach, as a non-pharmacological intervention, intends to alter the brain activity by providing the feedback of EEG activity (Loo \& Makeig, 2012). NFB increases the beta activity and suppresses the theta activity, and has been observed to improve SA in some disorders, such as reading disorder (Mosanezhad Jeddi \& Nazari, 2013) and attention-Deficit/Hyperactivity Disorder (ADHD) (Steiner, Frenette, Rene, Brennan, \& Perrin, 2014). 
As noted, SLD is often accompanied by impairments in neurocognitive functioning, such as deficits in SA (Lawrence, Ross, Hoffmann, Garavan, \& Stein, 2003). Recent studies have reported the positive effect of CR (Gray et al., 2012; De Giglio et al., 2015) and NFB (Mosanezhad Jeddi \& Nazari, 2013) on SA in SLD and some other disorders. However, no study has compared the effect of the $\mathrm{CR}$ and NFB training in children with SLD. Therefore, using a Randomized Controlled clinical Trial (RCT), this study aimed to determine and compare the efficacy of CR and NFB on SA among the elementary school students with SLD.

\section{Materials \& Methods}

\subsection{Study design and participants}

In this preliminary RCT, 53 elementary students with the diagnosis of SLD, according to the DSM-5 criteria (American Psychiatric Association, 2013), were selected. The participants were in the age range of 7 to 10 years and were attending the Learning Disability Center (Tabriz, IR Iran). They were randomly assigned in three study groups: NFB ( $\mathrm{n}=18), \mathrm{CR}(\mathrm{n}=18)$, and control group $(\mathrm{n}=17)$.

Before study enrolment, a psychologist screened the participants for study eligibility. The inclusion criteria were as follows: the age range of 7 to 10 years; the educational grade of 1 to 4; one or more years of education; no comorbidity of the other neurodevelopmental disorders, such as ADHD, communication disorder, Developmental Coordination Disorder (DCD), and autism spectrum disorder; the lack of other mental disorders, such as anxiety disorders, mood disorders, bipolar disorders; and the absence of conditions that could interfere with the diagnosis of SLD, according to the parents' reports and the recorded psychiatric cases. The exclusion criteria were abnormal mental ability, the chronic use of medications, receiving CR or NFB before entering the study, and unwillingness for cooperation.

\subsection{Intervention}

\subsubsection{Cognitive rehabilitation}

The training involved 20 sessions of CR three times per week (each session lasting 45 minutes). The CR is a tripartite model that combines interventions derived from several approaches: brain injury rehabilitation, educational psychology, and child clinical psychology (Butler $\&$ Copeland, 2002). Specific CR training was programmatic but individualized. The CR has several interdepen- dent components: hierarchically graded massed practice, strategy acquisition, and cognitive-behavioral intervention. The selected students completed a modified version of the attention process training cognitive rehabilitation program. This program was developed by Sohlberg and colleagues (Sohlberg, Johnson, Paule, Raskin, \& Mateer, 1999; Sohlberg \& Mateer, 1999). It is designed to strengthen the dimensions of attentional processes. The intervention for the preliminary RCT described here was identical to Butler and Copeland's CR approach (Butler \& Copeland, 2002). A certified CR training coach who was completely independent of the study team supervised the $\mathrm{CR}$ training.

\subsubsection{Neurofeedback}

The participants received 20 sessions of in-school NFB training for 30 to 45 minutes, two times per week; the sessions were monitored by a trained Research Assistant (RA). The RAs received a two-week standardized training to administer the NFB sessions. They filled out a standardized checklist for each participant at every session to monitor the implementation fidelity.

The specific NFB system (play attention, unique logic, and technology, fletcher, NC) detects two frequency ranges: one in the low-frequency theta brainwave range (4-8 Hz), and another in the high-frequency beta brainwave range (12-15 Hz). These brainwaves were measured using three EEG sensors: embedded in a standard bicycle helmet centrally located on the top of the skull, a grounding sensor, and a reference on the chin straps located bilaterally on the mastoids. Through the practices, participants learn to manipulate the figures on the computer screen, resulting in the suppression of the theta and increase of the beta waves activity. As the theta-beta ratio changes, the algorithm of the computer program scores the participants, thus, they gradually learn to improve attention on six different tasks.

\subsection{Measurements}

\subsubsection{Continuous performance test}

The SA was examined using a standard computerized version of the Conners continuous performance test (CPT) (Version 3.0; Conners, 1994). During the sequential presentation of a series of letters ( 1 inch in size), the subject was instructed to press the keyboard for every letter except $X$. In this test, each letter appears on the screen for $250 \mathrm{~ms}$; this task takes approximately 14 minutes to complete. Inattention is assessed by the number of omission errors (ie, the targets to which the participant did 
not respond), commission errors (i.e. false positives) and response times (Adams, Roberts, Milich, \& Fillmore, 2011; López-Martín, Fernández-Jaén, \& Carretié, 2013). Some studies have reported an acceptable validity of CPT for the research objectives (Raz, Bar-Haim, Sadeh, \& Dan, 2014). In this study, the reliability coefficients for the CPT were an adequate value of 0.78 .

\subsection{Outcome assessment}

The participants of the study groups were evaluated for SA using CPT. The SA was assessed at the baseline and one month after the beginning of the study. Accordingly, the participants in the experimental groups were evaluated before and after 20 sessions of CR or 20 sessions of NFB, while the participants of the control group were evaluated at the baseline and one month after the entry, without any intervention.

\subsection{Statistical analysis}

Data were presented as mean \pm standard deviation and frequency (percentage). The Multivariate Analysis of Covariance (MANCOVA) followed by the Bonferroni post hoc test was used for the comparison of the variables, between three study groups and before and after the interventions. Also, the effect sizes (estimated as Cohen's F2) were provided. The effect size values were rated as small, medium, and large for the F2 value of $0.02,0.15$, and 0.35 , respectively (Cohen, 1992). All statistical analyses were conducted with the IBM SPSS-20 (IBM Corp, 2011). The P values of less than 0.05 were considered statistically significant.

\section{Results}

\subsection{Demographic data}

A total of 69 students with SLD were recruited and screened for eligibility (Figure 1). Of them, 16 students did not meet the eligibility criteria: six showed the presence of attention-deficit/hyperactivity disorder and or DCD, based on the parents' reports and the recorded psychiatric cases; four received NFB, and two received CR before entering the study, and four were over 11 years of age. The remaining 53 children were randomized to one of the three study groups. At the Pre-test, three children of each CR and NFB training groups were dropped out. Also, two children of the control group were dropped out at the Post-test. Therefore, 45 children completed the study: the CR $(n=15)$, NFB $(n=15)$, and control $(n=15)$ groups.

The total sample included 60 individuals, wherein 24 participants $(40 \%)$ were boys and 36 participants $(60 \%)$ were girls. Besides, $5 \%, 33.3 \%$, and $61.7 \%$ of the participants were in the age ranges of 7 to 8 years, 8 to 9 years, and 9 to 10 years, respectively. The mean $\pm \mathrm{SD}$ age in the CR, NFB, and control groups were $8.66 \pm 1.48$ years, $8.40 \pm 1.73$ years, and $8.53 \pm 1.63$ years, respectively. Moreover, 16 students $(51.7 \%$ ( were enrolled in the first-grade elementary school, 26 students (43.3\%) were enrolled in the second-grade elementary school, two students $(3.3 \%)$ were enrolled in the third-grade elementary school, and one student $(1.7 \%)$ were enrolled in the fourth-grade elementary school. No statistically

Table 1. Descriptive statistics of the CPT variables at the pre-test and post-test, by the study group

\begin{tabular}{|c|c|c|c|}
\hline \multirow{2}{*}{ SA } & \multirow{2}{*}{ Groups } & \multicolumn{2}{|c|}{$\mathrm{M} \pm \mathrm{SD}$} \\
\hline & & Pre-test & Post-test \\
\hline \multirow{3}{*}{ Omissions } & CR & $2.05 \pm 4.26$ & $1.06 \pm 1.43$ \\
\hline & NFB & $1.43 \pm 3.25$ & $0.82 \pm 2.60$ \\
\hline & Control & $1.16 \pm 3.36$ & $1.28 \pm 3.93$ \\
\hline \multirow{3}{*}{ Commissions } & CR & $1.53 \pm 9.73$ & $1.42 \pm 4.20$ \\
\hline & NFB & $1.35 \pm 8.60$ & $0.98 \pm 5.40$ \\
\hline & Control & $9.06 \pm 1.48$ & $9.85 \pm 1.84$ \\
\hline \multirow{3}{*}{ Response time } & CR & $34.01 \pm 434.56$ & $30.98 \pm 332.40$ \\
\hline & NFB & $31.55 \pm 453.73$ & $29.60 \pm 347.53$ \\
\hline & Control & $32.23 \pm 423.30$ & $32.57 \pm 436.21$ \\
\hline
\end{tabular}


Table 2. Comparison of the cpt variables at the pre-test and post-test, between the three study groups

\begin{tabular}{cccc}
\hline CA & F & P & ES (d) \\
\hline Omissions & 3.07 & 0.035 & 0.14 \\
Commissions & 3.81 & 0.015 & 0.17 \\
Response time & 10.23 & 0.001 & 0.36 \\
\hline
\end{tabular}

ES: Effect Size (Cohen's d); TP: Test Power

NEUR SCIENCE

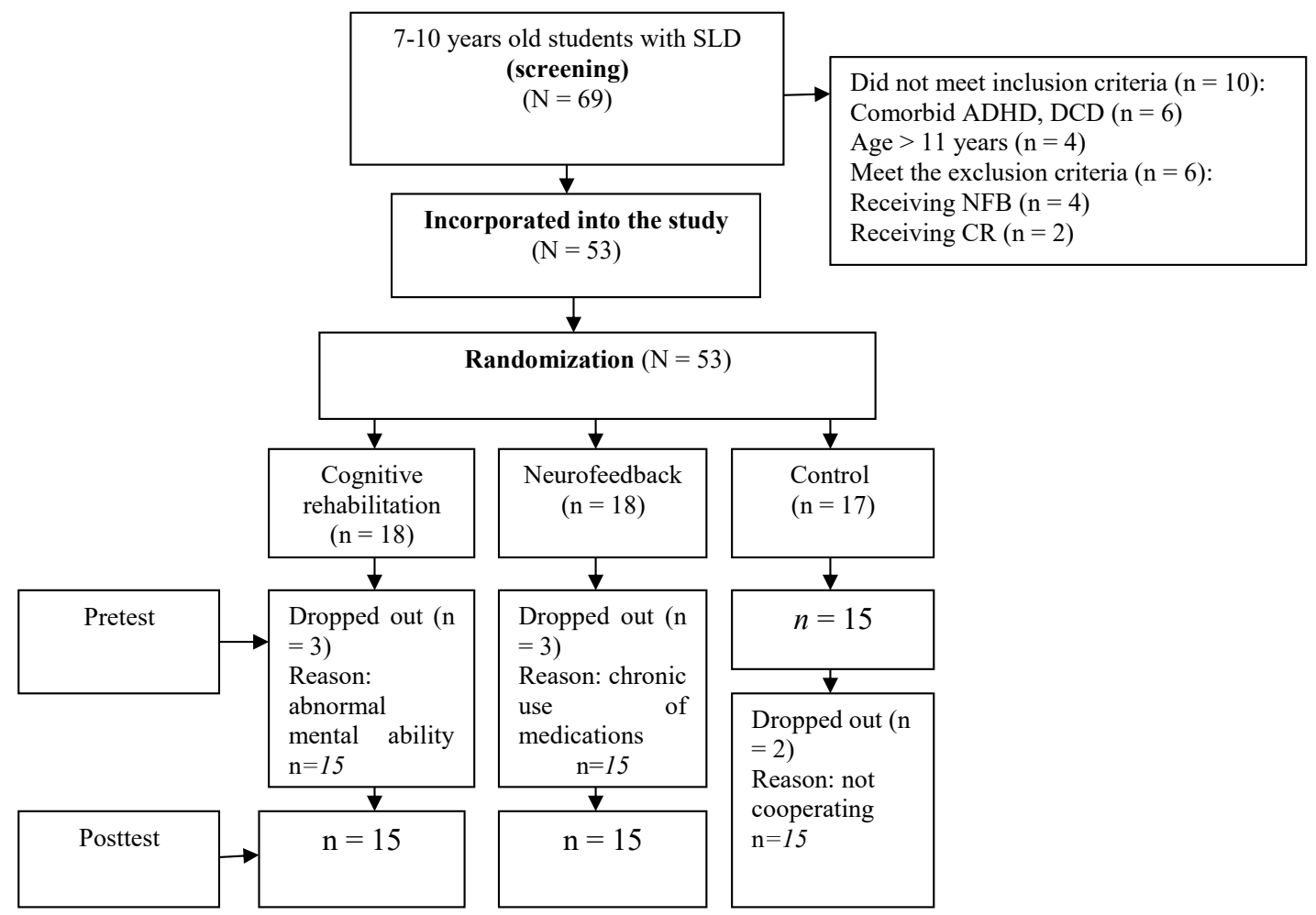

Figure 1. Distribution of the study students

significant difference was found between the three study groups in terms of demographic variables $(\mathrm{P}<0.05)$.

\subsection{CPT}

Table 1 presents the evaluated variables of CPT between the three groups, at the Pre-test and Post-test.

All the measures of the omission errors, commission errors, and response time improved from the pre to Post-test, in the intervention groups but not in the control group (Table 1).

Table 2 represents the comparison of the CPT variables at the pre-test and post-test, between the three study groups.

The MANCOVA showed a statistically significant difference at the $\mathrm{P}<0.05$ level between the study groups in omission errors $\left(\mathrm{F}_{3,53}=3.07, \mathrm{P}=0.035\right)$, commission errors $\left(\mathrm{F}_{3,53}=3.81, \mathrm{P}=0.015\right)$, and response time $\left(\mathrm{F}_{3,53}=10.23\right.$, $\mathrm{P}=0.001)$. The eta squared statistic for the scores of the omission errors and commission errors $(0.14$ and 0.17 , respectively) indicated a medium effect size. However, the eta squared statistic of the response time score (0.36) indicated a large effect size (Table 2).

The post hoc comparisons with the Bonferroni test indicated a significant effect for the omission errors, commission errors, and response time, with the higher scores of the CR group than that of the NFB group $(\mathrm{P}<0.001)$. Also, there was a significant effect for omission errors, commission errors, and response time, with the higher scores of the experimental groups than that of the control group $(\mathrm{P}<0.001)$. 


\section{Discussion}

Placebo in the present study caused no significant effect The analysis of data revealed some changes in the SA of the NFB and CR groups that included students with SLD. For example, the omission errors, commission errors, and response time of the CPT decreased after the NFB and CR training.

Different research studies indicated that children with SLD have deficits in SA as important correlates of executive functioning (Willcutt et al., 2011). The improvement of these abilities could result in growth in academic skills among people with LD (Swanson \& Jerman, 2007).

Mosanezhad Jeddi, and Nazari (2013) reported the successful use of NFB for continuous attention and working memory improvements; the NFB decreased theta and enhanced beta waves $(15-18 \mathrm{~Hz})$ in people with a reading disorder. Furthermore, some studies reported the efficacy of NFB on the improvement of SA in ADHD (Steiner, Frenette, Rene, Brennan, \& Perrin, 2014). Despite these reports regarding the useful effects of NFB on SA, Rostami et al. (2017) showed that NFB did not affect CA in patients with traumatic brain injury. This contradiction is justified by the various disorders discussed in both studies. Also, their study suffered from the reduction of the sample size.

The main finding of our study is that compared with NFB, the CR program reduced omission errors, commission errors, and response time, as assessed by CPT.

Some studies evaluated the effect of CR on cognitive functions, such as the attention of the people with SLD. In a randomized controlled trial study, Gray et al. (2012) evaluated the effect of a computerized working memory training program on adolescents with LD/ADHD. They compared the attention of these people before and after the math training and working memory training. Finally, they showed that working memory training is more effective than math training on attention. Compared with previous studies, the effect sizes of the omission errors, commission errors, and especially response time were more than those found by Gray et al. (2012).

Furthermore, CR can significantly affect sustained attention (Rodríguez-Blanco, Lubrini, Vidal-Mariño, \& Ríos-Lago, 217). For example, Kasper et al. (2015) reported the improvements of SA after the CR training, in people with Alzheimer disease. Some other studies replicated these findings in people with other diseases, including cerebral palsy (Muriel, Garcia-Molina, Apari-
cio-Lopez, Ensenat, \& Roig-Rovira, 2014) and multiple sclerosis (Amato et al., 2013; De Giglio et al., 2015).

The underlying mechanisms of the clinical improvement observed in our students with SLD following the $\mathrm{CR}$ are difficult to identify. The $\mathrm{MMRI}$-based studies reported a functional reorganization in specific brain regions following the rehabilitative programs that focused on SA or executive functions (Sastre-Garriga, Alonso, Renom, \& Arévalo, 2011; Cerasa et al., 2013). Also, a structural reorganization has been described even after a short CR intervention period (Cerasa et al., 2013). We hypothesize that similar mechanisms were involved in the present study population; however, the functional and structural modifications associated with CR training programs have remained to be investigated.

Our findings presented that the NFB protocol improved the scores of the Conners CPT, and the training protocol was set to reduce the theta activity $(4-8 \mathrm{~Hz})$ and increase the beta activity $(12-15 \mathrm{~Hz})$. The different kinds of neurofeedback will have different outcomes. If we change the location or frequency of the sensors, it will change to a very different protocol (Marzbani, Marateb, \& Mansourian, 2016). Therefore, NFB may be more effective than the $\mathrm{CR}$ training, but the confirmation of this will require more research in the future.

The CR is more effective than the NFB on SA improvement, among students with SLD. However, we believe that for making an exact decision about the use of CR in students with SLD, more research projects with follow-up should be done.

\section{Ethical Considerations}

\section{Compliance with ethical guidelines}

All ethical principles are considered in this article. The participants were informed about the purpose of the research and its implementation stages; they were also assured about the confidentiality of their information; moreover, they were free to leave the study whenever they wished, and if desired, the research results would be available to them.

\section{Funding}

This research did not receive any grant from funding agencies in the public, commercial, or non-profit sectors. 


\section{Authors' contributions}

All authors were equally contributed in preparing this article.

Conflict of interest

The authors declare that they have no conflict of interest

\section{References}

Adams, Z.W., Roberts, W.M., Milich, R., \& Fillmore, M.T. (2011). Does response variability predict distractibility among adults with attention-deficit/hyperactivity disorder? Psychol Assess, 23, 427-36. [DOI:10.1037/a0022112] [PMID] [PMCID]

Amato, M.P., Langdon, D., Montalban, X., Benedict, R.H., DeLuca, J., Krupp, L.B., et al. (2013). Treatment of cognitive impairment in multiple sclerosis: Position paper. Journal of Neurology, 260(6),1452-1468. [DOI:10.1007/s00415-012-66780] [PMID]

American Psychiatric Association. (2013) Diagnostic and Statistical Manual of Mental Disorders, $5^{\text {th }}$ Edition. New York: American Psychiatric Association.

Bergera, T., \& Cassutob, H. (2014). The effect of environmental distractors incorporation into a CPT on sustained attention and ADHD diagnosis among adolescents. Journal of Neuroscience Methods, 222, 62-8. [DOI:10.1016/j.jneumeth.2013.10.012] [PMID]

Play Attention [Internet] 2013. [Updated: 2013 Aug 22] Available from: www.playattention.com.

Butler, R. W., \& Copeland, D. R. (2002). Attentional processes and their remediation in children treated for cancer: A literature review and the development of a therapeutic approach. Journal of the International Neuropsychology Society, 8(1), 115-24. [DOI:10.1017/S1355617701020112]

Cerasa, A., Gioia, M.C., Valentino, P., Nisticò, R., Chiriaco, C., Pirritano, D., et al. (2013). Computer-assisted cognitive rehabilitation of attention deficits for multiple sclerosis: a randomized trial with FMRI correlates. Neurorehabil Neural Repair, 27(4), 284-95. [DOI:10.1177/1545968312465194] [PMID]

Cohen, J. (1992). A power primer. Psychol Bull, 112, 155-9. [DOI:10.1037/0033-2909.112.1.155] [PMID]

Conners, C. K. (1994). The Conners Continuous Performance Test. Toronto: Multi-Health Systems. https://www.pearsonclinical.co.uk/Psychology/ChildMentalHealth/ChildADDADHDBehaviour/conners-cpt3/conners-continuous-performance-test-third-edition.aspx

Daniel, S. S., Walsh, A. K., Goldston, D. B., Arnold, E. M., Reboussin, B. A., \& Wood, F. B. (2006). Suicidality, school dropout, and reading problems among adolescents. Journal of Learning Disabilities, 39(6), 507-14. [DOI:10.1177/00222194060 390060301] [PMID]

De Giglio, L., De Luca, F., Prosperini, L., Borriello, G., Bianchi, V., \& Pantano, P., et al. (2015). A low-cost cognitive reha- bilitation with a commercial video game improves sustained attention and executive functions in multiple sclerosis: A pilot study. Neurorehabil Neural Repair, 29(5), 453-61. [DOI:10.1177/1545968314554623] [PMID]

Duncan, C. C., Rumsey, J. M, Wilkniss, S. M., Denckla, M. B., Hamburger, S. D., \& Odou-Potkin M. (1994). Developmental dyslexia and attention dysfunction in adults: brain potentia indices of information processing. Psychophysiology, 31(4), 386-401. [DOI:10.1111/j.1469-8986.1994.tb02447.x] [PMID]

Facoetti, A., \& Molteni, M. (2000). Is attentional focusing an inhibitory process at distractor location? Cognitive Brain Research, 10, 185-8. [DOI:10.1016/S0926-6410(00)00031-8]

Facoetti, A., Zorzi, M., Cestnick, R., Lorusso, M. L., \& Molteni, M., et al. (2006). The relationship between visuo-spatial attention and non-word reading in developmental dyslexia. Cogn Neuropsychol, 23, 841-55. [DOI:10.1080/02643290500483090] [PMID]

Galletly, C., \& Rigby, A. (2013). An overview of cognitive reme-diation therapy for people with severe mental illness. International Scholarly Research Notices, 2013, 984932. [DOI:org/10.1155/2013/984932]

Gray, S. A., Chaban, P., Martinussen, R., Goldberg, R., Gotlieb, H., Kronitz, R., et al. (2012). Effects of a computerized working memory training program on working memory, attention, and academics in adolescents with severe LD and comorbid ADHD: A randomized controlled trial. Journal of Child Psychology and Psychiatry, 53(12), 1277-84. [DOI:10.1111/j.14697610.2012.02592.x] [PMID]

Kasper, E, Ochmann, S., Hoffmann, W., Schneider, W., Cavedo, E., \& Hampel H., et al. (2015). Cognitive rehabilitation in alzheimer's disease - a conceptual and methodological review. Journal of Prevention of Alzheimer's Disease, 2, 142-52. [DOI: 10.14283/jpad.2015.58]

Lawrence, N. S., Ross, T.J., Hoffmann, R., Garavan, H., \& Stein, E.A. (2003). Multiple neuronal networks mediate sustained attention. Journal of Cognitive Neuroscience, 15(7), 1028-38. [DO I:10.1162/089892903770007416] [PMID]

Loo, S.K., \& Makeig, S. (2012). Clinical utility of EEG in attentiondeficit/ hyperactivity disorder: a research update. Neurotherapeutics, 9, 569-87. [DOI:10.1007/s13311-012-0131-z] [PMID] [PMCID]

López-Martín, S., Albert, J., Fernández-Jaén, A., \& Carretié L. (2013). Emotional distraction in boys with ADHD: Neural and behavioral correlates. Brain and Cognition, 83, 10-20. [DOI:10.1016/j.bandc.2013.06.004] [PMID]

Marzbani, H., Marateb, H. R., \& Mansourian, M. (2016). Neurofeedback: A comprehensive review on system design, methodology and clinical applications. Basic and Clinical Neuroscience, 7(2), 143-58. [DOI:10.15412/J.BCN.03070208] [PMID] [PMCID]

Marzocchi, G.M., Ornaghi, S., \& Barboglio, S. (2009). What are the causes of the attention deficits observed in children with dyslexia? Child Neuropsychology, 15(6), 567-81 [DOI:10.1080/09297040902740660] [PMID]

Moll, K., Kunze, S., Neuhoff, N., Bruder, J., \& Schulte-Korne, G. (2014). Specific learning disorder: Prevalence and gender differences. PLoS One, 9(7), 1-8. [DOI:10.1371/journal. pone.0103537] [PMID] [PMCID] 
Jeddi, E. M., \& Nazari, M. A. (2013). Effectiveness of EEG-Biofeedback on attentiveness, working memory and quantitative electroencephalography on reading disorder. Iranian Journal of Psychiatry and Behavioral Sciences, 7(2), 35-43. [PMCID] [PMID]

Muriel, V., Garcia-Molina, A., Aparicio-Lopez, C., Ensenat, A., \& Roig-Rovira, T. (2014). Cognitive stimulation in children with cerebral palsy. Revista de Neurología, 59(10), 443-8. [DOI:10.33588/rn.5910.2014095]

Nazari, M.A., Querne, L., De Broca, A., \& Berquin, P. (2012) Effectiveness of EEG biofeedback as compared with methylphenidate in the treatment of attention-deficit/hyperactivity disorder: A clinical out-come study. Neuroscience \& Medicine, 2(2), 78-86. [DOI:10.4236/nm.2011.22012]

Oken, B. S., Salinsky, M. C., \& Elsas, S. M. (2006). Vigilance, alertness, or sustained attention: physiological basis and measurement. Clinical Neurophysiology, 117(9), 1885-901. [DOI:10.1016/j.clinph.2006.01.017] [PMID] [PMCID]

Peijnenborgh, J. C., Hurks, P. M.,A.P., Vles J. S., \& Hendriksen, J. G. (2016). Efficacy of working memory training in children and adolescents with learning disabilities: A review study and meta-analysis. Neuropsychological Rehabilitation, 26(5-6), 645-72. [DOI:10.1080/09602011.2015.1026356] [PMID]

Raz, S., Bar-Haim, Y., Sadeh, A., \& Dan, O. (2014). Reliability and validity of the online continuous performance test among young adults. Assessment, 21(1), 108-18. [DOI:10.1177/1073191112443409] [PMID]

Rodríguez-Blanco, L., Lubrini., G, Vidal-Mariño, C., \& RíosLago, M. (2017). Efficacy of cognitive rehabilitation of attention, executive functions, and working memory in psychotic disorders: A systematic review. Actas Españolas de Psiquiatría, 45(4), 167-78. [PMID]

Rostami, R., Salamati, P., Yarandi, K. K., Khoshnevisan, A., Saadat, S., \& Kamali, Z. S., et al. (2017). Effects of neurofeedback on the short-term memory and continuous attention of patients with moderate traumatic brain injury: A preliminary randomized controlled clinical trial. Chinese Journal of Traumatology, 20(5), 278-82. [DOI:10.1016/j.cjtee.2016.11.007] [PMID] [PMCID]

Sastre-Garriga, J., Alonso, J., Renom, M., \& Arévalo, M. J. (2011). A functional magnetic resonance proof of concept pilot trial of cognitive rehabilitation in multiple sclerosis. Multiple Sclerosis Journal, 17, 457-67. [DOI:10.1177/1352458510389219] [PMID]

Shapiro, E. S., \& Lentz, F. E. (1991). Vocational-technical programs: follow-up of students with learning disabilities. Exceptional children, 58(1), 47-59. [DOI:10.1177/00144029910580 0105]

Sohlberg, M., \& Mateer, C. A. (1999). Attention Process Training I (APT-I). Wake Forest, NC: Lash \& Associates.

Sohlberg, M. M., Johnson, L., Paule, L., Raskin, S.A, \& Mateer, C. A. (1999). Attention Process Training II (APT-II). Wake Forest, NC: Lash \& Associates.

Steiner, N. J., Frenette, E. C., Rene, K. M., Brennan, R. T., \& Perrin E. C. (2014). In-school neurofeedback training for ADHD: Sustained improvements from a randomized control trial. Pediatrics, 133(3), 483-92. [DOI:10.1542/peds.2013-2059] [PMID]

Sterr, A. M. (2004). Attention performance in young adults with learning disabilities. Learning and Individual Differences, 14, 125-33. [DOI:10.1016/j.lindif.2003.10.001]
Swanson, H.L, \& Jerman, O. (2007). The influence of working memory on reading growth in subgroups of children with reading disabilities. Journal of Experimental Child Psychology, 96(4), 249-83. [DOI:10.1016/j.jecp.2006.12.004] [PMID]

Vogel, S. A., \& Reder, S. (1989). Educational attainment of edults with learning disabilities. In S. A. Vogel \& S. Reder (Eds.), Learning disabilities, literacy, and adult education. Baltimore, MD: Paul H. Brookes.

Willcutt, E. G., Boada, R., Riddle, M. W., Chhabildas, N., DeFries, J. C., \& Pennington, B. F. (2011). Colorado learning difficulties questionnaire: Validation of a parent-report screening measure. Psychological Assessment, 23(3), 778-91. [DOI:10.1037/ a0023290] [PMID] [PMCID] 